\section{Renal dosing of allopurinol results in suboptimal gout care}

We commend the authors of the '2016 updated EULAR evidence-based recommendations for the management of gout' for advocating starting allopurinol at a lower dose in patients with normal renal function. ${ }^{1}$ Specifically, this recognises an approach to potentially decrease the risk of precipitating flares of gout early in the course of urate lowering, and also to possibly decrease the risk of severe cutaneous reactions (SCARs) compared with higher starting doses of allopurinol. ${ }^{2}$ However, we note that the authors do not provide a recommendation on starting dose for patients with renal impairment, the patient group most likely to benefit from starting at a much lower dose of allopurinol. ${ }^{2}$

Furthermore, recommendation $\# 9,{ }^{\mathrm{i}}$ which advocates limiting the maximal dose of allopurinol based on creatinine clearance (CrCL), is concerning. It is well-documented that such practice results in suboptimal management of hyperuricaemia in the majority of patients with gout. ${ }^{3}$ Adhering to the CrCL-based allopurinol dosing has been a major contributing factor to the under-treatment of gout since this scheme was published in 1984. ${ }^{4}$ The authors acknowledge there are data to support careful dose escalation in those with renal impairment, ${ }^{5}{ }^{6}$ but then state that the low incidence of SCARs makes it difficult to quantify this risk and therefore advise against a dose-escalation approach. Allopurinol hypersensitivity syndrome/SCAR occurs early after starting allopurinol. ${ }^{2}{ }^{7}$ While renal impairment is a major risk factor for allopurinol hypersensitivity syndrome/ SCAR, there is no evidence that long-term restriction of allopurinol dose according to CrCL lowers this risk in patients who tolerate low starting doses of allopurinol. ${ }^{8}{ }^{9}$ The approach advocated by the European League Against Rheumatism panel could lead to a clinical situation where patients may be exposed to potential risks of allopurinol, without the benefits achieved by careful dose escalation to achieve serum urate target. By focusing on the maximum dose of allopurinol rather than the starting dose in patients with renal dysfunction, an important safety point has been missed and this may inadvertently reinforce fears of prior decades about allopurinol use in patients with renal dysfunction where more recent data ${ }^{5}$ has shown that allopurinol can be safely used in patients with renal impairment.

Recommendation \#9 is also likely to reduce the quality of care for the many people with gout in regions with limited access to urate-lowering therapies other than allopurinol. Furthermore, recommending febuxostat if renally dosed allopurinol is insufficient for gout management raises potential concern for patients with renal impairment. Studies examining the safety and efficacy of febuxostat in people with gout and comorbid renal impairment have been sparse, with 266 subjects with chronic kidney disease (CKD) stage 3 randomised to febuxostat in the CONFIRMS clinical trial, ${ }^{10}$ and only 19 subjects with CKD stage 4 randomised to febuxostat in another recent trial. ${ }^{11}$ This is important, as febuxostat itself has been associated with SCARs, including in patients with

${ }^{\mathrm{i}}$ Recommendation \#9: "In patients with renal impairment, the allopurinol maximum dosage should be adjusted to creatinine clearance. If the SUA target cannot be achieved at this dose, the patient should be switched to febuxostat or given benzbromarone with or without allopurinol, except in patients with estimated glomerular filtration rate $<30 \mathrm{~mL} / \mathrm{min}$." renal impairment and/or previous hypersensitivity to allopurinol, leading the European Medical Agency and Health Canada to issue warnings regarding this issue. ${ }^{12}{ }^{13} \mathrm{It}$ is presently not known if this represents a true cross-reactivity or rather an individual's propensity to experiencing a drug reaction.

The lack of clarity in the optimal management of hyperuricaemia in patients with gout and renal impairment should have also been considered an important topic for future research. Given that almost half of all people with gout suffer from moderate-to-severe CKD and the limited treatment options available in this setting, a major knowledge gap and unmet need remains the optimal means of managing these challenging presentations. We recognise that a large, well-designed study will likely be needed, considering the low incidence of allopurinol hypersensitivity syndrome (AHS)/SCAR reactions. Nonetheless, recommending dosing of allopurinol based on $\mathrm{CrCL}$ in the absence of evidence to support this practice will not improve the historically poor management of gout in patients with renal disease.

Tuhina Neogi, ${ }^{1}$ Nicola Dalbeth, ${ }^{2}$ Lisa Stamp, ${ }^{3}$ Geraldo Castelar, ${ }^{4}$ John Fitzgerald, ${ }^{5}$ Angelo Gaffo, ${ }^{6,7}$ Ted R Mikuls, ${ }^{8}$ Jasvinder Singh, ${ }^{7}$ Janitzia Vázquez-Mellado, ${ }^{9} \mathrm{~N}$ Lawrence Edwards ${ }^{10}$

${ }^{1}$ Clinical Epidemiology Research and Training Unit, Boston University School of Medicine, Boston, Massachusetts, USA

${ }^{2}$ Department of Immunology, University of Auckland, Auckland, New Zealand

${ }^{3}$ Department of Medicine, Otago University, Christchurch, Canterbury, New Zealand

${ }^{4}$ Department of Rheumatology, Pedro Ernesto University Hospital, Rio de Janeiro, Brazil

${ }^{5}$ Medicine/Rheumatology, David Geffen School of Medicine, University of California, Los Angeles, Los Angeles, California, USA

${ }^{6}$ Department of Medicine, Birmingham VA Medical Center, Birmingham, Alabama, USA

${ }^{7}$ Department of Medicine, University of Alabama at Birmingham, Birmingham, Alabama, USA

${ }^{8}$ Department of Medicine, University of Nebraska Medical Center, Omaha, Nebraska, USA

${ }^{9}$ Servicio de Reumatologia, Hospital General de Mexico, Mexico City, Mexico

${ }^{10}$ Department of Medicine, University of Florida College of Medicine, Gainesville, Florida, USA

Correspondence to Professor N Lawrence Edwards, University of Florida, Gainesville, FL 32610, USA; edwarn|@medicine.ufl.edu

Contributors All named authors of this letter participated in the development and wording of our communication.

Competing interests None declared.

Provenance and peer review Not commissioned; internally peer reviewed.

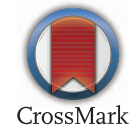

To cite Neogi T, Dalbeth N, Stamp L, et al. Ann Rheum Dis 2017;76:e1.

Received 11 August 2016

Accepted 12 August 2016

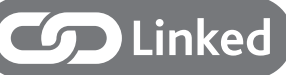

- http://dx.doi.org/10.1136/annrheumdis-2016-210356

Ann Rheum Dis 2017;76:e1. doi:10.1136/annrheumdis-2016-210352

REFERENCES

1 Richette P, Doherty M, Pascual E, et al. 2016 updated EULAR evidence-based recommendations for the management of gout. Ann Rheum Dis 2016; Published Online First 25 July 2016. 
2 Stamp LK, Taylor WJ, Jones PB, et al. Starting dose is a risk factor for allopurinol hypersensitivity syndrome: a proposed safe starting dose of allopurinol. Arthritis Rheum 2012;64:2529-36.

3 Dalbeth N, Kumar S, Stamp L, et al. Dose adjustment of allopurinol according to creatinine clearance does not provide adequate control of hyperuricemia in patients with gout. J Rheumatol 2006;33:1646-50.

4 Hande KR, Noone RM, Stone WJ. Severe allopurinol toxicity. Description and guidelines for prevention in patients with renal insufficiency. Am J Med 1984;76:47-56.

5 Stamp LK, O'Donnell JL, Zhang $M$, et al. Using allopurinol above the dose based on creatinine clearance is effective and safe in patients with chronic gout, including those with renal impairment. Arthritis Rheum 2011;63:412-21.

6 Rees F, Jenkins W, Doherty M. Patients with gout adhere to curative treatment if informed appropriately: proof-of-concept observational study. Ann Rheum Dis 2013;72:826-30.

7 Kim SC, Newcomb C, Margolis D, et al. Severe cutaneous reactions requiring hospitalization in allopurinol initiators: a population-based cohort study. Arthritis Care Res (Hoboken) 2013;65:578-84.
8 Dalbeth N, Stamp L. Allopurinol dosing in renal impairment: walking the tightrope between adequate urate lowering and adverse events. Semin Dial 2007;20:391-5.

9 Vázquez-Mellado J, Morales EM, Pacheco-Tena C, et al. Relation between adverse events associated with allopurinol and renal function in patients with gout. Ann Rheum Dis 2001;60:981-3.

10 Becker MA, Schumacher HR, Espinoza LR, et al. The urate-lowering efficacy and safety of febuxostat in the treatment of the hyperuricemia of gout: the CONFIRMS trial. Arthritis Res Ther 2010;12:R63.

11 Saag KG, Whelton A, Becker MA, et al. Impact of febuxostat on renal function in gout patients with moderate-to-severe renal impairment. Arthritis Res Ther 2016;68:2035-43.

12 Adenuric: EPAR—Product Information. http://www.ema.europa.eu/docs/en_GB/ document_library/EPAR___Product_Information/human/000777/WC500021812.pdf (accessed 27 Jul 2016).

13 Summary Safety Review_-ULORIC (febuxostat)—Assessing a possible risk of drug reaction/rash with eosinophilia and systemic symptoms (DRESS). 2016. http://www. hc-sc.gc.ca/dhp-mps/medeff/reviews-examens/uloric3-eng.php (accessed 27 Jul 2016). 